\title{
STRONG DIFFERENTIABILITY OF THE NORM AND ROTUNDITY OF THE DUAL
}

\author{
J. R. GILES \\ (Received 10 March; revised 27 October 1977) \\ Communicated by E. Strzelecki
}

\begin{abstract}
For normed linear spaces two similar characterizations of strong differentiability of the norm and rotundity of the dual space are established, but it is shown that in general there is no causal relation between these two concepts.
\end{abstract}

Subject classification (Amer. Math. Soc. (MOS) 1970): 46 B 10.

Given a real normed linear space $X$, and $y \in X$ and $r>0$ we denote by $B[y ; r]$ closed ball $\{x \in X:\|x-y\| \leqslant r\}$. We denote by $B(X)$ the closed unit ball $\{x \in X:\|x\| \leqslant 1\}$ and by $S(X)$ the unit sphere $\{x \in X:\|x\|=1\}$. Given $x \in S(X)$ we denote by $D(x)$ the set $\left\{f \in S\left(X^{*}\right): f(x)=1\right\} . X$ is said to be smooth at $x \in S(X)$ if $D(x)$ is a single point set. The norm of $X$ is said to be strongly differentiable (Fréchet differentiable) at $x \in S(X)$ if for some $f_{x} \in D(x)$,

$$
\lim _{y \rightarrow 0} \frac{\|x+y\|-\|x\|-f_{x}(y)}{\|y\|}=0 .
$$

If the norm of $X$ is strongly differentiable at $x \in S(X)$ then $X$ is smooth at $x$, (see Giles (1971), p. 109), but from Example 2 below it can be observed that the converse is not true even for reflexive spaces. We say that $X$ is rotund at $x \in S(X)$ if for any $y \in S(X) \backslash\{x\}, \frac{1}{2}(x+y) \notin S(X)$. It is well known that if $X^{*}$ is rotund on $S\left(X^{*}\right)$ then $X$ is smooth on $S(X)$, but it follows from a result of Klee (1959), p. 62, and Day's theorem given in Theorem 2(i) $\Leftrightarrow$ (ii) below that there exists a separable space which can be equivalently renormed so that $X$ is smooth on $S(X)$ and $X^{*}$ 
is not rotund on $S\left(X^{*}\right)$. We might well ask whether there is some tighter relation between strong differentiability of the norm and rotundity of the dual.

Phelps (1960), p. 977, explored an important geometrical implication of strong differentiability of the norm. His Theorem 2.2 motivates the characterization of strong differentiability of the norm which we give in Theorem 1 below.

Vlasov (1970), p. 776, showed rotundity of the dual to be a geometrical property with significant consequences. His Theorem 1 is a fascinating characterization of rotundity of the dual which suggests comparison with Phelp's investigation of strong differentiability of the norm. We localize Vlasov's theorem in Theorem 2 below to highlight its similarity with our characterization of strong differentiability of the norm.

To prove our first theorem we require the following elementary result.

LEMMA. For a normed linear space $X$, the norm is strongly differentiable at $x \in S(X)$ if for some $f_{x} \in D(x)$ and all $z \in X$ such that $f_{x}(z)=0$,

$$
\lim _{z \rightarrow 0} \frac{\|x+z\|-\|x\|}{\|z\|}=0 \text {. }
$$

Proof. Given $\varepsilon>0$ there exists a $0<\delta<\frac{1}{3}$ such that

$$
\frac{|\|x+z\|-\|x\||}{\|z\|}<\frac{1}{2} \varepsilon \text { when }\|z\|<3 \delta .
$$

Now any $y \in S(X)$ can be represented in the form $y=\alpha x+z$ where $\alpha=f_{x}(y)$ and $f_{x}(z)=0$. Since $\|z\| \leqslant 2$, when $|\lambda|<\delta$ we have

$$
\left\|\frac{\lambda z}{1+\alpha \lambda}\right\|<\frac{2 \delta}{1-\delta}<3 \delta
$$

and so

$$
\begin{aligned}
\left|\frac{\|x+\lambda y\|-\|x\|}{\lambda}-f_{x}(y)\right| & \leqslant 2 \frac{|\|x+[\lambda /(1+\alpha \lambda)] z\|-\|x\||}{|\lambda /(1+\alpha \lambda)|\|z\|} \\
& <\varepsilon \text { for all } y \in S(X) .
\end{aligned}
$$

THEOREM 1. For a normed linear space $X$, the norm is strongly differentiable at $x \in S(X)$ if and only if for some $f_{x} \in D(x)$ and for every bounded closed convex set $K$ where $\sup f_{x}(K)<1$, there exists an $n_{0}$ such that $K \subseteq B\left[\left(1-n_{0}\right) x ; n_{0}\right]$.

Proof. Suppose that the norm is strongly differentiable at $x$ but that for $f_{x} \in D(x)$ there exists a bounded closed convex set $K$ such that $\sup f_{x}(K)<1$ and for every $n$ there exists an $x_{n} \in K \backslash B[(1-n) x ; n]$. Writing $y_{n} \equiv x_{n} /(n-1)$, since $\left\{x_{n}\right\}$ is bounded, 
we have $y_{n} \rightarrow 0$. Now

$$
\frac{\left\|x+y_{n}\right\|-\|x\|}{\left\|y_{n}\right\|}=\frac{\left\|(1-n) x-x_{n}\right\|-\|(n-1) x\|}{\left\|x_{n}\right\|}>\frac{n-(n-1)}{\left\|x_{n}\right\|}=\frac{1}{\left\|x_{n}\right\|} .
$$

But

$$
\frac{f_{x}\left(y_{n}\right)}{\left\|y_{n}\right\|}=\frac{f_{x}\left(x_{n}\right)}{\left\|x_{n}\right\|} \leqslant \frac{k}{\left\|x_{n}\right\|}, \quad \text { where } k \equiv \sup f_{x}(K)<1 .
$$

Again since $\left\{x_{n}\right\}$ is bounded we conclude that this contradicts the strong differentiability of the norm at $x$.

Conversely, suppose that the norm of $X$ is not strongly differentiable at $x \in S(X)$. Then from the lemma we see that for any $f_{x} \in D(x)$ there exists an $r>0$ and a sequence $\left\{y_{n}\right\}$ where $f_{x}\left(y_{n}\right)=0$ and $y_{n} \rightarrow 0$ such that

$$
\frac{\left\|x+y_{n}\right\|-\|x\|}{\left\|y_{n}\right\|} \geqslant r \text { for all } n \text {. }
$$

We may assume that

$$
\frac{1}{n+1}<\left\|y_{n}\right\|<\frac{1}{n}
$$

For a given $n$, consider the Minkowskian plane associated with the normed linear subspace generated by $x$ and $y_{n}$. Denote by $a_{n}$ the point of intersection of the interval from 0 to $x+y_{n}$ with $S(X)$. Denote by $d_{n}$ the point of intersection of the

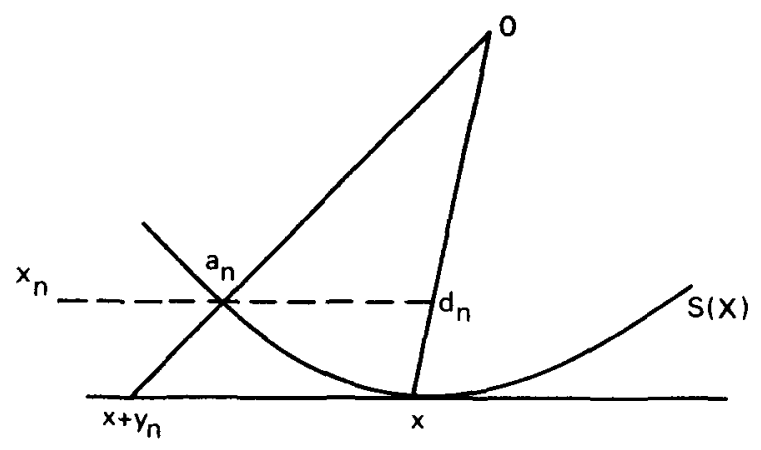

line through $a_{n}$ parallel to the line through $x$ and $x+y_{n}$, with the interval from 0 to $x$. On the ray from $d_{n}$ through $a_{n}$ choose a point $x_{n}$ outside $B(X)$ such that $\left\|x_{n}-a_{n}\right\|=1 / n$. Consider the similarity transformation $z \mapsto z^{\prime}=n z-(n-1) x$ taking $B(X)$ to $B[(1-n) x ; n]$. It is clear that

$$
\left\|x-d_{n}\right\|=\frac{\left\|x+y_{n}\right\|-\|x\|}{\left\|x+y_{n}\right\|} \geqslant \frac{1}{2}\left(\left\|x+y_{n}\right\|-\|x\|\right) \geqslant \frac{1}{2} r\left\|y_{n}\right\|>\frac{1}{2} r\left(\frac{1}{n+1}\right),
$$


and so

$$
\left\|x-d_{n}^{\prime}\right\|=n\left\|x-d_{n}\right\|>\frac{1}{4} r .
$$

But also

$$
\left\|x-d_{n}\right\|=\frac{\left\|x+y_{n}\right\|-\|x\|}{\left\|x+y_{n}\right\|}<\left\|y_{n}\right\|<\frac{1}{n}
$$

and so

$$
\left\|x_{n}^{\prime}-x\right\|=n\left\|x_{n}-x\right\|<3
$$

Therefore, in $X$ the closed convex set $K \equiv \overline{c o}\left\{x_{n}^{\prime}\right\}$ has the properties that $\sup f_{x}(K)<1$ and $K$ is bounded but for each $n, x_{n}^{\prime} \in K \backslash B[(1-n) x ; n]$.

We present our local characterization of rotundity of the dual through a localized form of Day's characterization of rotundity of the dual (Day (1973), p. 145).

Theorem 2. For a normed linear space $X$ with dual space $X^{*}$, given $f \in S\left(X^{*}\right)$, the following statements are equivalent.

(i) The dual space $X^{*}$ is rotund at $f$.

(ii) For any $g \in S\left(X^{*}\right) \backslash\{ \pm f\}$, writing $L \equiv f^{-1}(0) \cap g^{-1}(0)$, and for any sequence $\left\{x_{n}\right\}$ in $S(X)$ where $f\left(x_{n}\right) \rightarrow 1$, the two-dimensional factor space $X / L$ is smooth at $Y$ where $Y$ is a cluster point of $\left\{x_{n}+L\right\}$ in $X / L$.

(iii) For any sequence of closed balls $\left\{B_{n}\right\}$, where $B_{n} \equiv B\left[z_{n} ; R_{n}\right]$ and $B_{n} \subseteq B_{n+1}$ for all $n$ and $R_{n} \rightarrow \infty$, contained in the half-space $\{y \in X: f(y) \leqslant 1\}$, there exists an $r \leqslant 1$ such that $\overline{U B}_{n}=\{y \in X: f(y) \leqslant r\}$.

PrOOF. (ii) implies (i). Suppose that $X^{*}$ is not rotund at $f$. Then there exists a $g \in S\left(X^{*}\right) \backslash\{f\}$ such that $\frac{1}{2}(f+g) \in S\left(X^{*}\right)$. Write $L \equiv f^{-1}(0) \cap g^{-1}(0)$ and consider the two-dimensional factor space $X / L$. Define the linear functionals $F$ and $G$ on $X / L$ by

$$
F(x+L)=f(x) \text { and } \quad G(x+L)=g(x) \text { for all } x \in X .
$$

Now $\|F\|=\|f\|=1$ and $\|G\|=\|g\|=1$. There exists a sequence $\left\{x_{n}\right\}$ in $S(X)$ such that $(f+g)\left(x_{n}\right) \rightarrow\|f+g\|=2$ and so $f\left(x_{n}\right) \rightarrow 1$ and $g\left(x_{n}\right) \rightarrow 1$. Now the set $\left\{x_{n}+L\right\}$ is contained in $B(X / L)$ and so it has a cluster point $Y$ in $B(X / L)$. We may suppose that $x_{n}+L \rightarrow Y$. But

$$
F\left(x_{n}+L\right)=f\left(x_{n}\right) \rightarrow 1 \quad \text { and } \quad G\left(x_{n}+L\right)=g\left(x_{n}\right) \rightarrow 1,
$$

and so $F(Y)=1$ and $G(Y)=1$, and we conclude that $X / L$ is not smooth at $Y$. 
(iii) implies (ii). Suppose that there exists a $g \in S\left(X^{*}\right) \backslash\{ \pm f\}$ such that, writing $L \equiv f^{-1}(0) \cap g^{-1}(0)$, for some sequence $\left\{x_{n}\right\}$ in $S(X)$ where $f\left(x_{n}\right) \rightarrow 1$ we have that the two-dimensional factor space $X / L$ is not smooth at $Y$ where $Y$ is a cluster point of $\left\{x_{n}+L\right\}$ in $X / L$. Then there exist linearly independent $F$ and $G \in S\left((X / L)^{*}\right)$ such that $F(Y)=1$ and $G(Y)=1$. Consider a real decreasing sequence $\left\{\varepsilon_{n}\right\}$ such that $\varepsilon_{n} \rightarrow 0$ and a sequence of closed balls $\left\{\widetilde{B}_{n}\right\}$ in $X / L$ where $\widetilde{B}_{n} \equiv \widetilde{B}\left[(1-n) Y ; n-\varepsilon_{n}\right]$. It is clear that $\sup F\left(\widetilde{B}_{n}\right) \leqslant 1$ and $\sup G\left(\widetilde{B}_{n}\right) \leqslant 1$. Now for each $n$ it is possible to find $z_{n} \in(1-n) Y$ such that

$$
\left\|z_{n+1}-z_{n}\right\|<1+\varepsilon_{n}-\varepsilon_{n+1} .
$$

Consider the sequence of closed balls $\left\{B_{n}\right\}$ in $X$ where $B_{n} \equiv B\left[z_{n} ; n-\varepsilon_{n}\right]$. It is clear that $B_{n} \subseteq B_{n+1}$ for all $n$. But also for any $n$ and $x \in B_{n}$ we have that $x+L \in \widetilde{B}_{n}$ and so

$$
f(x)=F(x+L) \leqslant 1 \text { and } g(x)=G(x+L) \leqslant 1 .
$$

Therefore, $\overline{U B}_{n} \subseteq\{y \in X: f(y) \leqslant 1\} \cap\{y \in X: g(y) \leqslant 1\}$ and so $\overline{U B}_{n}$ is not a half-space.

(i) implies (iii). Suppose that there exists a sequence of closed balls $\left\{B_{n}\right\}$, where $B_{n} \equiv B_{n}\left[z_{n} ; R_{n}\right]$ and $B_{n} \subseteq B_{n+1}$ for all $n$ and $R_{n} \rightarrow \infty$, contained in the half-space $\{y \in X: f(y) \leqslant 1\}$ such that $\overline{U B}_{n}$ is not a half-space. Then there exists a $z \in \operatorname{int}\{y \in X: f(y) \leqslant 1\}$ and $z \notin \overline{U B}_{n}$. Now $\overline{U B}_{n}$ is a convex set with an interior point and so there exists a $g \in S\left(X^{*}\right)$ such that $g(z) \geqslant \sup g\left(\overline{U B}_{n}\right)$. Clearly, $f$ and $g$ are linearly independent. We may assume that $\sup f\left(\overline{U B}_{n}\right)=\sup g\left(\overline{U B}_{n}\right)=1$. Writing

$$
\varepsilon_{n} \equiv 1-\sup f\left(B_{n}\right) \text { and } \delta_{n} \equiv 1-\sup g\left(B_{n}\right) \text {, }
$$

we have that $\varepsilon_{n}, \delta_{n} \rightarrow 0$. Now

$$
f\left(z_{n}\right)=1-\varepsilon_{n}-R_{n} \text { and } g\left(z_{n}\right)=1-\delta_{n}-R_{n} \text { for all } n \text {. }
$$

Consider the sequence $\left\{x_{n}\right\}$ where $x_{n} \equiv\left(z_{1}-z_{n}\right) / R_{n}$. Since $B_{n} \subseteq B_{n+1}$ for all $n$ we have that $x_{n} \in B(X)$ for all $n$. Now

$$
\begin{aligned}
f\left(x_{n}\right) & =\frac{f\left(z_{1}\right)-f\left(z_{n}\right)}{R_{n}} \\
& =\frac{1-\varepsilon_{1}-R_{1}-1+\varepsilon_{n}+R_{n}}{R_{n}} \\
& =1+\frac{\varepsilon_{n}-\varepsilon_{1}-R_{1}}{R_{n}} \rightarrow 1 \text { as } n \rightarrow \infty .
\end{aligned}
$$

Similarly $g\left(x_{n}\right) \rightarrow 1$. So then $(f+g)\left(x_{n}\right) \rightarrow 2$ and we have that $\|f+g\|=2$ and we conclude that $X^{*}$ is not rotund at $f$. 
It is obvious that a normed linear space $X$ is smooth at $x \in S(X)$ if and only if for some $f_{x} \in D(x), \overline{U B}[(1-n) x ; n]=\left\{y \in X ; f_{x}(y) \leqslant 1\right\}$. This condition is clearly weaker than those given in the above theorems.

These two theorems, characterizing strong differentiability of the norm and rotundity of the dual in terms of increasing sequences of balls, prompt investigation of the relation between the two concepts.

In a finite dimensional space $X_{n}$ the norm is strongly differentiable at $x \in S\left(X_{n}\right)$ if the dual space is rotund at $f_{x} \in D(x)$. However, the converse is not true locally.

EXAmple 1. Consider $R^{2}$ where, for $x \equiv\left(\lambda_{1}, \lambda_{2}\right),\|x\|_{\infty}=\max \left\{\left|\lambda_{1}\right|,\left|\lambda_{2}\right|\right\}$. Now $e_{1} \equiv(1,0)$ is a smooth point on the unit sphere and since the space is finite dimensional it is a point where the norm is strongly differentiable. The dual space is isometrically isomorphic to $R^{2}$ where, for $f \equiv\left(\mu_{1}, \mu_{2}\right),\|f\|_{1}=\left|\mu_{1}\right|+\left|\mu_{2}\right|$. Now $f_{1} \equiv(1,0) \in D\left(e_{1}\right)$ but the dual space is clearly not rotund at $f_{1}$.

For reflexive spaces the converse is true globally. However, it is possible to have a reflexive space whose norm is not strongly differentiable at a point of its unit sphere but which has rotund dual. The dual of this example was discussed in Giles (1976), p. 407.

EXAMPLE 2. Consider Hilbert space $l_{2}$ with its natural basis $\left\{e_{n}\right\}$. Brown (1974), p. 146 , has shown that it is possible to give $l_{2}$ an equivalent rotund norm such that for each $k \geqslant 2$ the two-dimensional subspaces $\operatorname{sp}\left\{e_{1}, e_{k}\right\}$ have an $l_{k}$-norm; that is, for $x \equiv\left\{\alpha_{1}, 0, \ldots, \alpha_{k}, 0, \ldots\right\}$,

$$
\|x\|=\left(\left|\alpha_{1}\right|^{k}+\left|\alpha_{k}\right|^{k}\right)^{1 / k} .
$$

Now $l_{2}$ with this norm is reflexive and its dual is smooth and is isometrically isomorphic to $l_{2}$ with norm such that for each $k \geqslant 2$ the two-dimensional subspaces $\operatorname{sp}\left\{e_{1}, e_{k}\right\}$ have $l_{k /(k-1)}$ norm; that is, for $x \equiv\left\{\alpha_{1}, 0, \ldots, \alpha_{k}, 0, \ldots\right\}$

$$
\|x\|=\left(\left|\alpha_{1}\right|^{k /(k-1)}+\left|\alpha_{k}\right|^{k /(k-1)}\right)^{1-1 / k} .
$$

In this space consider the sequence $\left\{y_{k+1}\right\}$ where

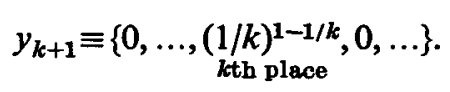

Now

$$
\left\|y_{k+1}\right\|=(1 / k)^{1-1 / k} \rightarrow 0 \text { as } k \rightarrow \infty
$$

and

$$
\left\|e_{1}+y_{k+1}\right\|=(1+1 / k)^{1-1 / k} \geqslant 1=\left\|e_{1}\right\| \text { for all } k \geqslant 2,
$$

so for $f_{e_{1}} \in D\left(e_{1}\right)$ we have

$$
f_{e_{1}}\left(y_{k+1}\right)=0 \text { for all } k \geqslant 2 .
$$


But

$$
\frac{\left\|e_{1}+y_{k+1}\right\|-\left\|e_{1}\right\|}{\left\|y_{k+1}\right\|}=(k+1)^{1-1 / k}-1+0 \text { as } k \rightarrow \infty .
$$

We conclude that the dual of Brown's space has norm not strongly differentiable at $e_{1}$.

The only relation left to explore is the global situation for a non-reflexive space $X$ with norm strongly differentiable on $S(X)$. Surely such a space does not necessarily have rotund dual. However, the construction of a counter-example would seem to involve a refinement of the construction given by Klee (1959), p. 62, to produce a smooth space with non-rotund dual. Such a construction could prove to be quite complicated.

\section{References}

A. L. Brown (1974), "A rotund reflexive space having a subspace of codimension two with a discontinuous metric projection", Michigan Math. J. 21, 145-151.

M. M. Day (1973), Normed Linear Spaces, 3rd ed. (Springer).

J. R. Giles (1971), "On a characterization of differentiability of the norm of a normed linear space”, J. Austral. Math. Soc. 12, 106-114.

J. R. Giles (1976), "Uniformly weak differentiability of the norm and a condition of Vlasov", J. Austral. Math. Soc. 21,(A) 393-409.

V. L. Klee (1959), "Some new results on smoothness and rotundity in normed linear spaces", Math. Ann. 139, 51-63.

R. R. Phelps (1960), "A representation theorem for bounded convex sets", Proc. Amer. Math. Soc. 11, 976-983.

L. P. Vlasov (1970), "Almost convex and Chebychev sets", Math. Notes Acad. Sci. USSR 8, 776-779.

University of Newcastle

N.S.W. 2308

Australia
University of Washington

Seattle, WA 98195

U.S.A. 